\title{
HbA1c measurements from dried blood spots: validation and patient satisfaction
}

\author{
M. Rebecca Fokkema ${ }^{1, *}$, Andries J. Bakker, \\ Fokje de Boer ${ }^{2}$, Jeltsje Kooistra ${ }^{1}$, Sifra de \\ Vries $^{1}$ and Albert Wolthuis ${ }^{1}$ \\ ${ }^{1}$ Stichting Klinisch Chemisch Laboratorium, Medical \\ Center Leeuwarden, Leeuwarden, The Netherlands \\ 2 Stichting Klinisch Chemisch Laboratorium, Hospital \\ De Sionsberg, Dokkum, The Netherlands
}

\begin{abstract}
Background: This study evaluates $\mathrm{HbA} 1 \mathrm{c}$ measurements from dried blood spots collected on filter paper and compares $\mathrm{HbA} 1 \mathrm{c}$ from filter paper (capillary blood) with $\mathrm{HbA} 1 \mathrm{c}$ measured in venous blood.

Methods: Patient satisfaction was evaluated using a questionnaire. The performance with the filter paper method was assessed by comparing $\mathrm{HbA} 1 \mathrm{c}$ results from EDTA-blood samples obtained via dried blood spots with $\mathrm{HbA} 1 \mathrm{c}$ results obtained with freshly hemolyzed blood (routine $\mathrm{HbA} 1 \mathrm{c}$ ). Adult patients visiting the outpatient clinic for $\mathrm{HbA} 1 \mathrm{c}$ analyses were recruited for the evaluation of dried blood spot sampling at home. Laboratory personnel collected a capillary blood sample on filter paper as well as a venous EDTA-blood sample. The participants collected another capillary blood sample at home and sent the dried filter paper back to the laboratory. Samples were analyzed with an immunoturbidimetric assay.

Results: Between-filter coefficient of variation was $1.8 \%$. Filter paper $\mathrm{HbA} 1 \mathrm{c}$ increased slightly during storage, particularly during the first 5 days. Filter paper $\mathrm{HbA} 1 \mathrm{c}$ highly correlated with routine $\mathrm{HbA} 1 \mathrm{c}$ $(r=0.987)$. The evaluation of samples collected at home showed comparable $\mathrm{HbA1c}$ values by filter paper and routine sampling methods $(n=93)$. Eightythree percent of participants said they would like the filter method to be brought into practice.

Conclusions: Home HbA1c sampling on filter paper is an acceptable sampling alternative for analysis of $\mathrm{HbA} 1 \mathrm{c}$.

Clin Chem Lab Med 2009;47:1259-64.
\end{abstract}

Keywords: capillary blood; dried blood spots; filter paper; HbA1c; patient satisfaction.

*Corresponding author: Dr. M.R. Fokkema, Universitair Medisch Centrum Groningen, Department of Laboratory Medicine, CMC-V, Room Y2.119, HPA EA61, Hanzeplein 1 9713 GZ Groningen, The Netherlands

Phone: + 31503615118

E-mail: m.r.heiner@lc.umcg.nl

Received March 12, 2009; accepted July 6, 2009;

previously published online September 14, 2009

\section{Introduction}

Diabetes mellitus is a major health problem worldwide. In 2007, it was estimated that 246 million adults $(7.3 \%)$ have diabetes mellitus, with $85 \%-$ 95\% having type 2 diabetes. European and North American regions have the highest prevalence at $8.4 \%$ and $9.2 \%$, respectively (1). Tight glycemic control of patients with diabetes mellitus is important since it reduces the risk of diabetic complications, including microvascular, neuropathic $(2,3)$ and macrovascular (4) complications. Glycemic control is monitored by measurement of $\mathrm{HbA} 1 \mathrm{c}$ content. HbA1c is the main glycated hemoglobin fraction, formed by the irreversible binding of glucose to the $\mathrm{N}$-terminal valine of the $\beta$-chains of normal adult hemoglobin (HbA). HbA1c reflects mean blood glucose concentrations over the previous 1-2 months, and forms an integral part of diabetes monitoring $(5,6)$. It is recommended that HbA1c be measured every 6 months in patients with stable glycemic control, and every 3 months during changes in treatment or for those who do not meet treatment goals (5). The American Diabetes Association recommends maintaining $\mathrm{HbA} 1 \mathrm{c}$ as close to normal $(<6 \%)$ without significant hypoglycemia as optimal (5). However, many official institutes recommend an $\mathrm{HbA} 1 \mathrm{c}$ level below $7 \%$ as being desirable for adults (6). HbA1c is generally measured when the patient visits his or her physician for evaluation of treatment. Most laboratory $\mathrm{HbA} 1 \mathrm{c}$ assays are too laborious to provide fast $\mathrm{HbA} 1 \mathrm{c}$ results, and results are usually reported after the patient has visited his doctor. Home sampling devices allow HbA1c samples to be collected, transported and analyzed a few days before a patient visits the physicians' office. These methods allow patients to take their own capillary blood at home, and this eliminates a visit to the laboratory before visiting the physician. Potential devices for collection are EDTA cups or filter paper. Filter paper is easy to handle, can be sent in flat envelopes and is inexpensive. An alternative to home sampling devices is point-of-care testing (POCT). Unlike POCT, filter $\mathrm{HbA} 1 \mathrm{c}$ is measured by the same analytical method as measurement performed with venous EDTAblood for HbA1c. This helps avoid bias between analytical methods and eliminates the need for periodic method comparison studies. This study compares HbA1c from dried capillary blood on filter paper, taken by laboratory personnel and by patients themselves, with $\mathrm{HbA} 1 \mathrm{c}$ from freshly hemolyzed venous EDTA-blood samples. We also evaluated the satisfaction of patients with this home HbA1c sampling procedure. 


\section{Patients and methods}

\section{Laboratory evaluation protocol}

Filters, samples and analytical methods Optimal elution conditions, precision and stability for up to 5 days for $\mathrm{HbA} 1 \mathrm{c}$ obtained from dried blood collected on filter papers and the comparability with routine $\mathrm{HbA} 1 \mathrm{c}$ analysis was investigated. Filter paper was obtained from Roche Diagnostics (HbA1c via Post ${ }^{\circledR}$, Roche Diagnostics GmbH, Mannheim, Germany). Additional stability studies of up to 10 days were investigated with a second type of filter: Whatman $903^{\circledR}$ specimen collection paper (Whatman Nederland BV, Den Bosch, The Netherlands). The Whatman 903 collection paper is imprinted with five half-inch circles and has a wraparound cover to protect the paper before and after sample collection. EDTAblood samples from anonymized patients were selected based on previously measured $\mathrm{HbA} 1 \mathrm{c}$ concentrations. EDTAblood was applied to the filter paper and allowed to dry at room temperature for at least $3 \mathrm{~h}$. Stored samples were placed in a dark environment. Discs, $3 \mathrm{~mm}$ in diameter, were punched out of the filter paper, put in hemolyzing buffer (cat nr. 1488457, Roche, 1 disc per $250 \mu \mathrm{L}$ ) and were analyzed with the immunoturbidimetric Tinaquant ${ }^{\circledR} \mathrm{HbA} 1 \mathrm{c}$ assay on a MODULAR P-analyzer (Roche Diagnostics $\mathrm{GmbH}$, Mannheim, Germany). Routine HbA1c measurements were performed by analyzing a mixture of $10 \mu \mathrm{L}$ EDTA-blood with $1000 \mu \mathrm{L}$ hemolyzing buffer with the Tinaquant ${ }^{\circledR} \mathrm{HbA} 1 \mathrm{c}$ assay.

The initial laboratory evaluations (precision and stability) employed a $24 \mathrm{~h}$ elution period at $4^{\circ} \mathrm{C}$, according to elution conditions reported by Jeppsson (7). To improve the logistical processes in our laboratory, we also investigated the possibility of eluting filters for 1, 2, $3 \mathrm{~h}$ at room temperature. Filters for studies performed following this evaluation were eluted for $1 \mathrm{~h}$ at room temperature (additional stability studies, method comparison and home sampling evaluation).

Elution conditions Ten EDTA-samples were selected and spotted on Post ${ }^{\circledR}$ filters. Discs were punched out, placed in hemolyzing buffer and eluted for $24 \mathrm{~h}$ at $4^{\circ} \mathrm{C}$, or for 1,2 or $3 \mathrm{~h}$ at room temperature prior to $\mathrm{HbA} 1 \mathrm{c}$ analyses. A minimal elution period of $1 \mathrm{~h}$ was previously reported by Jeppsson (7) using the same filter and elution buffer.

Precision For precision studies, 20 samples were analyzed in duplicate by the routine method, or in duplicate from two Roche filter papers to calculate within- and between-filter precision. Calculations were performed according to CLSI EP5 (8). All discs were eluted for $24 \mathrm{~h}$ at $4^{\circ} \mathrm{C}$, according to elution conditions reported by Jeppsson (7).

Stability Based on the stability studies of Jeppsson (7), we initially employed a limited study protocol to ensure the stability of $\mathrm{HbA} 1 \mathrm{c}$ from dried blood spots. We analyzed blood spots from 10 patients (Roche filters) after storage for 0,1 and 5 days in the dark at room temperature. Discs were subsequently punched out and left to elute for $24 \mathrm{~h}$ at $4^{\circ} \mathrm{C}$ prior to analyses.

Additional stability studies Stability for up to 10 days was investigated using Whatman $903^{\circledR}$ filter paper. We analyzed blood spots from 10 patients (two filter papers per patient) stored for $0,1,5,7,8,9$ and 10 days at room temperature. To investigate the influence of exposure of the filter papers to air, one filter paper from each patient was stored exposed to air and the other was stored in a sealed plastic bag. Discs were punched out and eluted for $1 \mathrm{~h}$ at room temperature prior to analysis.

Method comparison We compared HbA1c concentrations using the routine method and using dried blood spots from filter paper. We analyzed the HbA1c concentrations of all EDTA samples collected during one day in our hospital laboratory using the routine whole blood hemolysate and blood collected on filter paper (Roche). Discs were eluted for $1 \mathrm{~h}$ at room temperature prior to analysis.

\section{Evaluation of home sampling and patient satisfaction}

Participants were recruited from patients visiting the outpatient clinic for HbA1c analysis. Patients aged $\geq 18$ years and familiar with taking their own capillary blood samples for home glucose monitoring were eligible for participation. The study was approved by the Medical Ethics Committee of the Medical Center Leeuwarden (The Netherlands). Volunteers gave written informed consent prior to participation. Immediately following collection of a venous EDTA-blood sample for their routine $\mathrm{HbA} 1 \mathrm{c}$ analysis, laboratory personnel collected a capillary blood sample which was applied to filter paper and allowed to dry for at least $3 \mathrm{~h}$. Participants were also asked to spot filter paper with capillary blood at home. For this, they received information on the proper procedure for blood collection and a package that contained a filter, envelope and written instructions. Participants were instructed to take a blood sample immediately after arrival at home, to let the filter dry for at least $3 \mathrm{~h}$ and to send it back to the laboratory in a sealed plastic cover that same day. On warm days $\left(>25^{\circ} \mathrm{C}\right)$, participants were instructed to post the sample a short time before the mailbox was emptied. Patients also received a questionnaire regarding their satisfaction with the procedure. EDTA-blood samples were analyzed on the day of sampling according to our routine procedure (see procedure above). Following arrival to the laboratory, filters were stored in sealed plastic covers in the dark at room temperature until analysis. All filters were analyzed within 10 days by placing one disc into $250 \mu \mathrm{L}$ hemolyzing buffer, and letting the sample elute for $1 \mathrm{~h}$ at room temperature.

\section{Data analysis}

Data were analyzed using the Analyse-It (Analyse-it software, Ltd., Leeds, UK) and Excel software (Microsoft Corporation, Redmond, WA, US). Precision was calculated using ANOVA [CLSI EP5 protocol (8)] and agreement with Passing and Bablok regression analysis [CLSI EP9 protocol (9)]. Student's t-test was used to investigate differences between groups and paired Student's t-test was used for differences between paired samples. Pearson's correlation coefficient was used to investigate the relationship between $\mathrm{HbA} 1 \mathrm{c}$ and any deviation from baseline. $\mathrm{p}<0.05$ was used for statistical significance.

\section{Results}

\section{Laboratory evaluation protocol}

Elution conditions The mean $\mathrm{HbA} 1 \mathrm{c}$ concentration of the selected EDTA samples was $7.2 \%$ (range $5.0 \%-9.3 \%, n=10$ ). Table 1 shows the relative deviation and the relationship between $\mathrm{HbA} 1 \mathrm{c}$ measured from filter paper and our routine $\mathrm{HbA} 1 \mathrm{c}$ method 
Table 1 Percentage deviation of HbA1c collected on filter paper from venous $\mathrm{HbA1c}$ concentrations and the relationship between filter paper and baseline venous HbA1c concentrations.

\begin{tabular}{lll}
\hline Elution condition & Bias, \% & Correlation \\
& Median [range $]$ & Pearson R \\
\hline $1 \mathrm{~h}$ at RT & $-1.4[-3.3,2.0]$ & 0.999 \\
$2 \mathrm{~h}$ at RT & $-2.9[-4.3,0.0]$ & 0.999 \\
$3 \mathrm{~h}$ at $\mathrm{RT}$ & $-3.2[-6.6,0.0]$ & 0.997 \\
$24 \mathrm{~h}$ at $4{ }^{\circ} \mathrm{C}$ & $-6.1[-11.0,-2.0]$ & 0.995 \\
\hline
\end{tabular}

Ten EDTA-blood samples were spotted and dried on filter paper and then eluted for 1, 2 and $3 \mathrm{~h}$ at room temperature, and for $24 \mathrm{~h}$ at $4^{\circ} \mathrm{C}$. The median [range] relative bias and correlation between filter paper and venous HbA1c is presented in the Table (filter - venous HbA1c). Relationships were evaluated using Passing and Bablok regression analyses and the Pearson correlation coefficient.

according to the different elution conditions. HbA1c concentrations from filter paper agreed most closely with HbA1c concentrations measured using our routine method when filter paper was eluted for $1 \mathrm{~h}$ at room temperature (RT).

Precision The 20 EDTA samples that we selected had a mean $\mathrm{HbA} 1 \mathrm{c}$ concentration of $7.2 \%$ (range $4.8 \%-10.9 \%$ ) when measured using our routine $\mathrm{HbA1c}$ method. The precision of duplicate $\mathrm{HbA1c}$ measurements using our routine analysis method was $1.1 \%$. Within-filter paper variation was $1.1 \%$ and between-filter variation was $1.8 \%$. Measurement of $\mathrm{HbA1c}$ quality control samples using our routine HbA1c analysis over a 2-month period showed between-run coefficients of variation of $1.6 \%(n=46$, mean $\mathrm{HbA} 1 \mathrm{c}$ concentration $=5.41 \%), 1.6 \% \quad(\mathrm{n}=46$, mean $=7.51 \%)$ and $1.3 \%(n=46$, mean $=10.65 \%)$.

Stability The first stability study showed that $\mathrm{HbA} 1 \mathrm{c}$ concentrations increased in the dried blood spots from days 0 to 5 (Figure 1). Filter paper HbA1c was lower compared with routine $\mathrm{HbA1c}$ measurements when analyzed on days 0 and $1(p<0.05)$, but not on day 5 .

Additional stability studies Stability results with the Roche filter paper were confirmed with additional stability studies using the Whatman $903^{\circledR}$ filter paper. HbA1c concentrations increased in dried blood spots from days 0 to 10, with the largest increases from day 0 to 5 (Figure 1). These changes resulted in a median

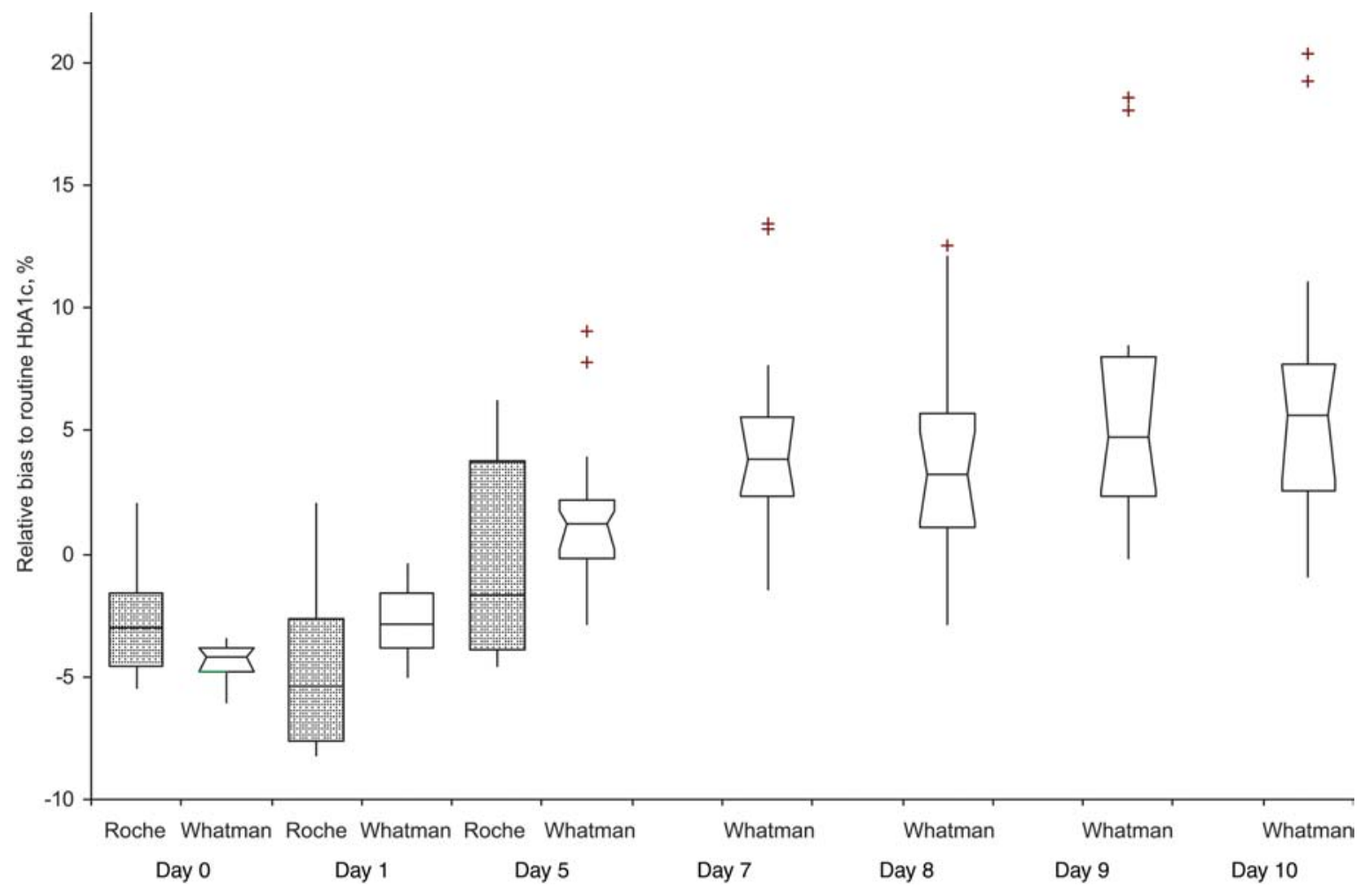

Figure 1 Stability studies.

The Figure displays boxplots of the relative bias to routine $\mathrm{HbA} 1 \mathrm{c}$ analysis of $\mathrm{HbA} 1 \mathrm{c}$ from dried blood spots stored up to 10 days in the dark at room temperature. Roche = primary stability study, 10 patients, filter papers stored for 0,1 and 5 days. Whatman =additional stability study, 10 patients (2 filter papers per patient), filter papers stored for $0,1,5,7,8,9,10$ days. The boxes represent medians and interquartile ranges. Outliers are shown as "+" and defined as observations which lie more than $1.5 \times$ the interquartile range (IQR) lower than the first quartile or $1.5 \times I \mathrm{R}$ higher than the third quartile. 
between-day variation of $3.5 \%$ [range $1.0 \%-8.0 \%$ (maximum 6.2\%, excluding outliers)]. Median between-day variation for filters stored $5-10$ days was $1.7 \%[0.7 \%-5.0 \%(0.7 \%-3.0 \%$, excluding outliers) $]$. Filter paper $\mathrm{HbA} 1 \mathrm{c}$ was lower than routine $\mathrm{HbA} 1 \mathrm{c}$ when analyzed on days 0 and 1 , and was higher on days 5-10 ( $p<0.05$ for all). Increases in HbA1c were similar for filters stored exposed to air compared with filters stored in a sealed plastic bag (all days $p>0.05$ ). A positive correlation was shown between routine $\mathrm{HbA} 1 \mathrm{c}$ measurements and the deviation in filter paper $\mathrm{HbA} 1 \mathrm{c}$ concentrations from routine $\mathrm{HbA} 1 \mathrm{c}$ concentrations during days 5-10, but not at days 0 and 1 . (Spearman correlation, $\mathrm{p}<0.05$ for all).

Method comparison The 73 samples had a mean $\mathrm{HbA} 1 \mathrm{c}$ concentration of $6.7 \%$ (range $4.7 \%-9.3 \%$ ) by routine $\mathrm{HbA} 1 \mathrm{c}$ analysis. $\mathrm{HbA} 1 \mathrm{c}$ concentrations measured using filter paper showed significant correlation with our routine $\mathrm{HbA} 1 \mathrm{c}$ method (Pearson $\mathrm{r}=0.987$ ). The intercept $[-0.011,95 \%$ confidence interval $(\mathrm{Cl})$ : -0.232 , +0.183] was not significantly different from zero, and the slope $(0.987,95 \% \mathrm{Cl}: 0.957,1.019)$ was not statistically different from 1 .

\section{Evaluation of home sampling and patient satisfaction}

The entire study group was comprised of 93 participants, 54 males and 39 females. The mean age was 59.1 years (range $23-85$ years). Five participants, two males and three females, did not return the home sampling filter paper, and four did not return the questionnaire. Since participants gave written informed consent, their $\mathrm{HbA} 1 \mathrm{c}$ concentrations measured using the laboratory filter paper and our routine method were included in the statistical analysis. Twenty-six percent of participants were reported to have diabetes mellitus type 1, 64\% type 2 and 10\% did not know their diabetes type. Participants had diabetes for $0.5-43$ years (median 8.0 years). Filter papers collected at home were analyzed within 10 days after sampling. All samples were sent when outdoor temperatures ranged from -9 to $11^{\circ} \mathrm{C}$, and were stored at $19-23^{\circ} \mathrm{C}$.

Figure 2 shows the comparison between the routine HbA1c method and the filter paper method, where "filter lab" indicates blood that was collected by laboratory personnel and "filter home" indicated blood that was collected at home. Passing and Bablok regression analysis revealed that both intercepts did not significantly deviate from zero and both slope values were not statistically different from 1. No significant bias was observed; +0.011 (95\% Cl: -0.037 , +0.060 ) between samples collected at home and $-0.025(-0.072,+0.021)$ samples collected in the lab with respect to measured $\mathrm{HbA} 1 \mathrm{c}$ concentrations. The Pearson correlation coefficients were 0.980 $(p<0.0001)$ for both comparisons. The Pearson correlation coefficient for the comparison between "filter lab" and "filter home" was $r=0.977(p<0.0001)$.
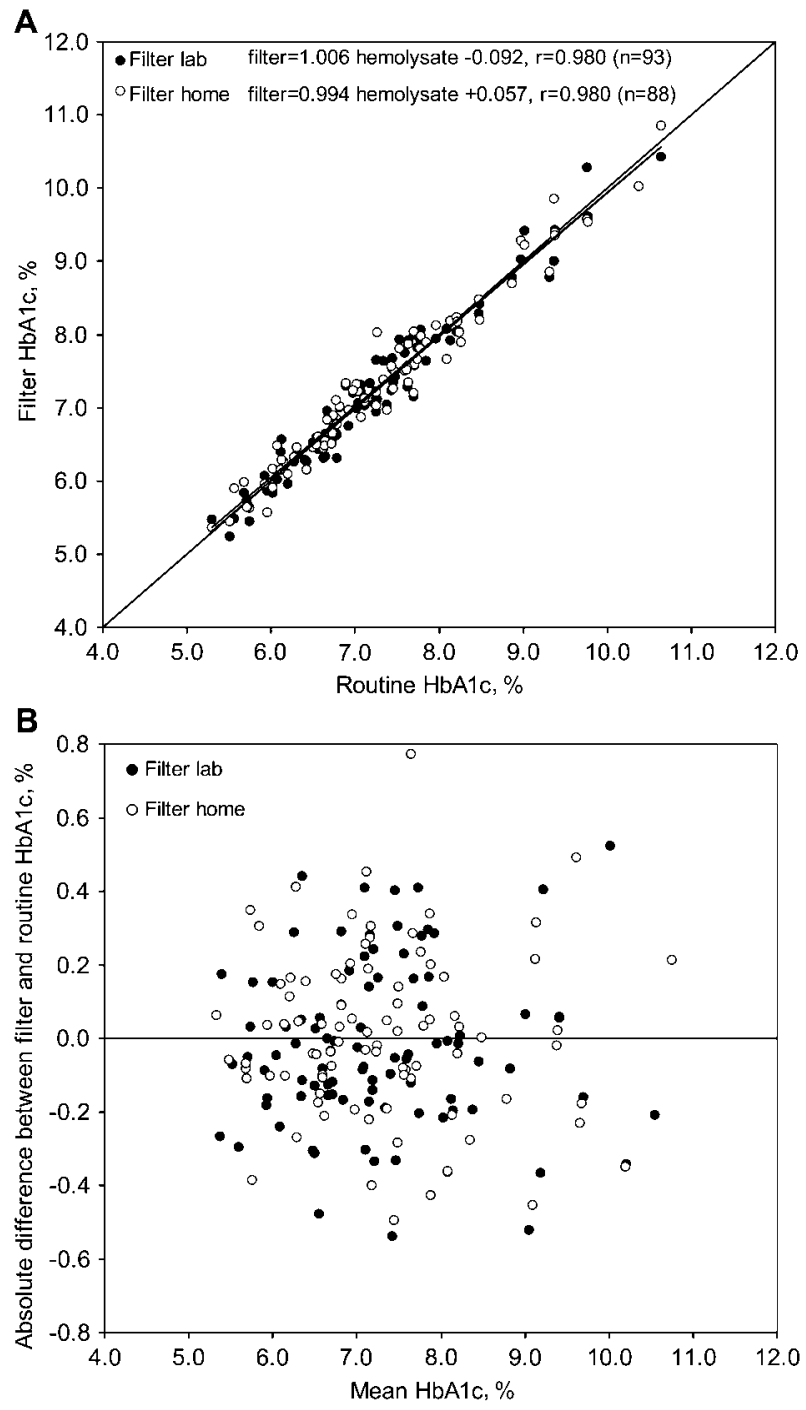

Figure 2 Passing and Bablok regression analysis (A) and bias plots (B) for home and lab filter paper HbA1c vs. HbA1c from hemolysate.

The dashed line in Figure 2A indicates perfect agreement between $x$ and $y$. The two regression lines for home and lab filter paper HbA1c are black lines. These are difficult to discriminate, since these overlap to a large extent.

The questionnaire revealed that many patients were satisfied with the filter paper method. Of the participants that responded, $90 \%$ indicated that the search for an alternative method for sampling is valuable, $94 \%$ indicated that the instructions for sampling were understandable, $97 \%$ said that collection with filter paper was easy, $96 \%$ were satisfied with home collection, $83 \%$ indicated that home collection in the future is desirable and $83 \%$ said that the filter paper method of collection should be brought in practice.

\section{Discussion and conclusions}

We evaluated the performance of dried blood spots for analysis of $\mathrm{HbA} 1 \mathrm{c}$ and investigated patient satisfaction with home collection of $\mathrm{HbA} 1 \mathrm{c}$ samples. The first report on measurement of glycated protein from 
dried blood spots was published in 1982 by Goldstein et al. (10). Since then, others have also demonstrated that $\mathrm{HbA} 1 \mathrm{c}$ can be measured using dried blood spots with acceptable accuracy and precision by use of ionexchange chromatography $(7,11-15)$, affinity chromatography $(9,13)$, a modified barbituric acid method (16) and by use of immunoturbidumetric assays (7, 17). The stability of $\mathrm{HbA} 1 \mathrm{c}$ in dried blood samples is the major concern. HbA1c may be formed by in vitro glycation of hemoglobin, and may also be degraded during storage in dried blood (18). Our results illustrate that pre-analytical factors need to be taken into account. In particular, storage and elution conditions were important factors. Storage increased $\mathrm{HbA} 1 \mathrm{c}$ concentrations, whereas prolonged elution times decreased $\mathrm{HbA} 1 \mathrm{c}$. Both effects may contribute significantly to the pre-analytical variation. It has been shown previously that high blood glucose concentrations increase glycated protein in dried blood samples (16). Our results confirm this finding, especially when one considers the positive relationship between $\mathrm{HbA} 1 \mathrm{c}$ concentrations and the changes in $\mathrm{HbA} 1 \mathrm{c}$ during storage. The increase may be greater at higher temperatures (14), although no temperature effect was evident in the study by Jeppsson et al. (12). The in vitro effects may also depend upon the analytical method that is used to measure HbA1c (18). Large clinical trials, such as the Diabetes Control and Complications Trial (2) and the United Kingdom Prospective Diabetes Study (3), have shown convincingly that an absolute $\mathrm{HbA} 1 \mathrm{c}$ difference of $1 \%$ is related to a significant reduction in risk from microvascular as well as macrovascular diabetic complications. Within a single patient, an absolute change of $1 \%$ is also considered to be significant. The within-person biological variation of $\mathrm{HbA} 1 \mathrm{c}$ in non-diabetic individuals is estimated to be very small. These biological factors demand strict analytical criteria. Currently, the recommended desirable goal for analytical precision is $<3 \%$, with an optimum goal of $<2 \%$ (19). Standardization of procedures and evaluation of the filter paper method in daily practise is therefore important. Another option is the use of filter paper pretreated (13-16, 20) with, for example, glucose oxidase. These have been shown to enhance the stability of $\mathrm{HbA} 1 \mathrm{c}$ when compared to untreated filter paper. The relative instability and subsequent variability did not seem to be a major problem when using filter paper in our daily practise, given the good agreement between home filter paper $\mathrm{HbA} 1 \mathrm{c}$ and routine $\mathrm{HbA} 1 \mathrm{c}$ analyses (Figure 2). In addition, filter paper $\mathrm{HbA} 1 \mathrm{c}$ analyzed between days 5 and 10 after collection had a median total analytical variation of $1.7 \%$, which is comparable to the analytical variation of routine $\mathrm{HbA} 1 \mathrm{c}$ analysis. This figure compares favorably to the $2.7 \%$ for 3-10 days storage of the pretreated filter papers that were stored with an unknown patented solution in the study of Parkes et al., who used a similar method of analysis (20).

A review of six studies (21) demonstrated that immediate availability of $\mathrm{HbA} 1 \mathrm{c}$ improves diabetes management, results in lower $\mathrm{HbA} 1 \mathrm{c}$ concentrations on subsequent visits and influenced clinical management in most studies. Kennedy et al. (22) performed a large randomized controlled study with 7893 adults and showed that point-of-care measurement of $\mathrm{HbA} 1 \mathrm{c}$ resulted in a significantly higher percentage of patients achieving $\mathrm{HbA} 1 \mathrm{c}$ concentrations below $7 \%$. Our study showed that many patients supported the search for an alternative method of collection, and were satisfied with the filter paper method tested. Patients are able to send their blood sample by post, and this method provides an opportunity for the physician to choose whether he or she wishes to examine stable patients during a hospital visit. We conclude that home collection of blood for $\mathrm{HbA} 1 \mathrm{c}$ on filter paper is an acceptable, easy and inexpensive alternative for collection of blood samples for $\mathrm{HbA} 1 \mathrm{c}$ analysis when pre-analytical factors are taken into account.

\section{Acknowledgements}

We gratefully acknowledge Harm de Vries and Monique Fortuin for the additional stability studies. We thank the laboratory technicians of the modular group for their support with $\mathrm{HbA} 1 \mathrm{c}$ measurements.

\section{References}

1. International Diabetes Federation. Facts and figures. http://www.idf.org/home/index.cfm?node = 264, 2009.

2. The Diabetes Control and Complications Trial Research Group. The effect of intensive treatment of diabetes on the development and progression of long-term complications in insulin-dependent diabetes mellitus. $\mathrm{N}$ Engl J Med 1993;329:977-86.

3. UK Prospective Diabetes Study (UKPDS) Group. Intensive blood-glucose control with sulphonylureas or insulin compared with conventional treatment and risk of complications in patients with type 2 diabetes (UKPDS 33). Lancet 1998;352:837-853.

4. Nathan DM, Cleary PA, Backlund JY, Genuth SM, Lachin JM, Orchard TJ, et al. Diabetes Control and Complications Trial/Epidemiology of Diabetes Interventions and Complications (DCCT/EDIC) Study Research Group. Intensive diabetes treatment and cardiovascular disease in patients with type 1 diabetes. N Engl J Med 2005;353: 2643-53.

5. American Diabetes Association. Standards of medical care in diabetes - 2006. Diabetes Care 2006;29:S4-S42.

6. Rutten GE, de Grauw WJ, Nijpels G, Goudswaard AN, Uitewaal PJM, van der Does FEE, et al. NHG-standard diabetes mellitus type 2. Tweede herziening. Huisarts Wet 2006;49:137-52.

7. Jeppsson JO. Determination of HbA1c by the Tina-quant HbA1c immunoassay using dried capillary blood on filter paper. Klin Lab 1993;39:1080-2.

8. National Committee for Clinical Laboratory Standards. Evaluation of Precision Performance of Clinical Chemistry Devices; approved guideline, NCCLS document EP5A (ISBN 1-56238-368-X). NCCLS, PA 1999.

9. National Committee for Clinical Laboratory Standards. Method comparison and bias estimation using patient samples; approved guideline. NCCLS document EP9-A (ISBN 1-56238-283-7). NCCLS, PA 1995.

10. Goldstein DE, Wiedmeyer HM, England JD, Little RR, Parker KM. Glycosylated protein in whole blood spotted on filter paper. Clin Chem 1982;28:386-7. 
11. Gay EC, Cruickshanks KJ, Chase HP, Klingensmith G, Hamman RF. Accuracy of a filter paper method for measuring glycosylated hemoglobin. Diabetes Care 1992;15: 108-10.

12. Jeppsson JO, Jerntorp P, Almër LO, Persson R, Ekberg G, Sundkvist G. Capillary blood on filter paper for determination of $\mathrm{HbA} 1 \mathrm{c}$ by ion exchange chromatography. Diabetes Care 1996;19:142-5.

13. Lomeo A, Bolner A, Scattolo N, Guzzo P, Amadori F, Sartori S, et al. HPLC analysis of HbA1c in dried blood spot samples (DBS): a reliable future for diabetes monitoring. Clin Lab 2008;54:161-7.

14. Eckerbom S, Bergqvist Y. Improved sample collection technique for capillary blood on filter paper for determination of glycated haemoglobin. Ann Clin Biochem 1989;26:148-50.

15. Little RR, Wiedmeyer HM, England JD, Knowler WC, Goldstein DE. Measurement of glycosylated wholeblood protein for assessing glucose control in diabetes: collection and storage of capillary blood on filter paper. Clin Chem 1985;31:213-6.

16. Eross J, Kreutzmann D, Jimenez M, Keen R, Rogers S, Crowell $\mathrm{C}$, et al. Colorimetric measurement of glycosylated protein in whole blood, red blood cells, plasma and dried blood. Ann Clin Biochem 1984;21:477-83.
17. Anjali, Geethanjali FS, Selva Kumar R, Seshadri MS. Accuracy of filter paper method for measuring glycated hemoglobin. J Assoc Physicians India 2007;55:115-9.

18. Eross J, Kreutzmann D, Crowell C, Silink M. Glycated haemoglobin measurement in dried blood on filter paper. Clin Chem 1986;32:2222.

19. Shephard MD. Analytical goals for point-of-care testing used for diabetes management in Australian health care settings outside the laboratory. Point of Care 2006;5: 177-185.

20. Parkes J, Ray R, Kerestan S, Davis H, Ginsberg B. Prospective evaluation of accuracy, precision, and reproducibility of an at-home hemoglobin A1c sampling kit. Diabetes Technol Ther 1999;1:411-9.

21. Winter WE. Does availability of hemoglobin A1c results at the point of care make a difference in the clinical care or outcome of the patient with diabetes? Point of Care 2006;5:58-61.

22. Kennedy L, Herman WH, Strange $P$, Harris A, GOAL AIC Team. Impact of active versus usual algorithmic titration of basal insulin and point-of-care versus laboratory measurement of $\mathrm{HbA1c}$ on glycemic control in patients with type 2 diabetes: the Glycemic Optimization with Algorithms and Labs at Point of Care (GOAL A1C) trial. Diabetes Care 2006;29:1-8. 